\title{
SABER DO FAMILIAR NA ADESÃO DA PESSOA HIPERTENSA AO TRATAMENTO: ANÁLISE COM BASE NA EDUCAÇÃO POPULAR EM SAÚDE ${ }^{1}$ FAMILY MEMBER'S KNOWLEDGE REGARDING HYPERTENSIVE PEOPLE'S ADHESION TO TREATMENT: AN ANALYSIS BASED ON A HEALTH EDUCATION PROGRAM SABER DEL FAMILIAR EN LA ADHESIÓN DE LA PERSONA HIPERTENSA AL TRATAMIENTO: ANÁLISIS CON BASE EN LA EDUCACIÓN POPULAR PARA LA SALUD
}

\author{
Klivia Regina de Oliveira Saraiva², Zélia Maria de Sousa Araújo Santos ${ }^{3}$, Fátima Luna Pinheiro Landim ${ }^{4}$, Amábili Couto Teixeira ${ }^{5}$
}

${ }^{1}$ Desenvolvido no Projeto "Fatores determinantes na adesão do usuário hipertenso ao tratamento: análise com enfoque na educação em saúde", financiado pela Fundação Cearense de Apoio ao Desenvolvimento Científico e Tecnológico (FUNCAP), Ministério da Saúde (MS) e Conselho Nacional de Desenvolvimento Científico e Tecnológico (CNPq).

${ }^{2}$ Enfermeira graduada pela Universidade de Fortaleza (UNIFOR). Mestranda em Enfermagem pela Universidade Federal do Ceará (UFC). Bolsista da Coordenação de Aperfeiçoamento de Nível Superior (CAPES). Membro do grupo de pesquisa Família, Ensino, Pesquisa e Extensão (FAMEPE) da UFC.

${ }^{3}$ Doutora em Enfermagem. Docente do Curso de Enfermagem e do Mestrado em Educação em Saúde da UNIFOR. Coordenadora de Enfermagem da Liga de Hipertensão Arterial do Hospital de Messejana (LHAHM). Líder do Núcleo de Estudo e Pesquisa na Promoção da Saúde de Pessoas com Alteração da Pressão Arterial (NUESPA).

${ }^{4}$ Doutora em Enfermagem. Docente do Curso de Enfermagem e do Mestrado em Educação em Saúde da UNIFOR.

${ }^{5}$ Acadêmica de Enfermagem da UNIFOR. Bolsista voluntária. Membro do NUESPA.

PALAVRAS-CHAVE:Família. RESUMO: Estudo exploratório-descritivo com o objetivo de analisar o saber do familiar na adesão Educação. Hipertensão. da pessoa hipertensa ao tratamento, realizado com 400 familiares de hipertensos inscritos na Liga de Hipertensão Arterial do Hospital de Messejana, em Fortaleza-CE. Os dados foram coletados através de entrevista, processados e analisados pelo programa Statistical Package for Social Science. A maioria dos familiares eram filhos e cônjuges com idade entre 40 a 49 anos, apresentavam noções sobre hipertensão e seu tratamento, tais como uso regular, número de tomadas diárias e tipos de medicamento, redução do sal e abstenção de gordura animal, e associavam como complicações infarto do miocárdio, acidente vascular cerebral e miocardiopatia. Conclui-se que o saber, embora superficial, era significativo na viabilização da adesão ao tratamento. Assim, inserir a família na problemática da hipertensão arterial, reporta-nos à importância de capacitá-la para investir na adesão da pessoa hipertensa ao tratamento.

KEYWORDS: Family. Education. Hypertension.

PALABRAS CLAVE: Familia. Educación. Hipertensión.

\begin{abstract}
This exploratory-descriptive study has the goal of analyzing the knowledge of family members of the hypertensive person in the adhesion to their treatment. It was carried out with the participation of 400 relatives of hypertensive persons enrolled in the League of Arterial Hypertension of the Hospital of Messejana, in Fortaleza, Ceará, Brazil. The data was collected through interviews, processed and analyzed by the Statistical Package is Social Science program. Most of the relatives were children and spouses aged between 40 to 49 years who presented notions of hypertension and its treatment, such as regular use, number of daily uptakes and medicine types, reduction of salt, and abstention of animal fat. They associated as complications myocardial arrest, cerebral vascular accidents, and extensive myocardiopathy. It is concluded that the knowledge, although superficial, was significant to the viability of adhesion to the treatment. Thus, involving the family in the issue of arterial hypertension emphasizes the importance of their qualification to invest in the hypertensive person's adhesion to their treatment.
\end{abstract}

RESUMEN: El presente artículo es un estudio exploratorio descriptivo que tiene como objetivo analizar el saber del familiar en la adhesión de la persona hipertensa al tratamiento. El estudio fue realizado con 400 familias de hipertensos inscritos en la Liga de Hipertensión Arterial del Hospital de Messejana, en la ciudad de Fortaleza - Ceará. Los datos fueron recolectados a través de entrevistas, procesados y analizados por el programa Statistical Package for Social Science. La mayoría de los familiares eran hijos o cónyuges entre 40 a 49 años de edad, los cuales poseían nociones sobre hipertensión y su tratamiento, tales como: el uso regular del medicamento, el número de tomadas diarias y los tipos de medicamento, la reducción de sal y la abstención en el consumo de grasa animal. También, asociaban como complicaciones, el infarto del miocardio, accidente vascular cerebral y miocardiopatía dilatada. A través de este estudio se concluye que el saber, aunque superficial, era significativo en la viabilidad de la adhesión al tratamiento. Entonces, la inclusión de la familia en la problemática de la hipertensión arterial, nos remite a la importancia de capacitarla para participar en la adhesión de la persona hipertensa al tratamiento.
Endereço: Klívia Regina de Oliveira Saraiva

Av. Dr. Joaquim Frota, 1278

60.833-430 - Água Fria, Fortaleza, CE.

E-mail: kliviaregina@yahoo.com.br
Artigo original: Pesquisa

Recebido em: 16/11/2006. Aprovação final: 09/04/2007. 


\section{INTRODUÇÃO}

A relação da educação com a promoção de saúde e a prevenção de doenças tem estado muito presente nas rodas científicas nos últimos dez anos. Muitos são os textos que abordam a temática, todos apontando para a necessidade dos profissionais da área da saúde se voltarem para o universo simbólico das pessoas, demarcado por padrões culturais em permanente processo de renovação.

Nesse contexto, as análises que consideram a família devem tomá-la como instituição responsável pela reprodução social, bem como pela transmissão de padrões culturais para ordenar a vida social, o que inclui a educação informal. É considerando essa inserção da família que, ao pensar na problemática da Hipertensão Arterial Sistêmica (HAS), o tema Educação Popular nos reporta à importância destas serem ouvidas quanto aos seus saberes, que possibilitam a adesão da pessoa hipertensa ao tratamento.

Cada família tem sua forma de "enxergar" as relações, bem como de enfrentar situações das mais variadas possíveis, e de sentir e perceber cada uma delas com suas singularidades.

Diante de uma doença crônica na família, como é o caso da HAS, ${ }^{1}$ esta se comporta de acordo com sua representação do que seja saúde-doença, evidenciando saberes diversos acerca da cura, que pode, inclusive, contrapor-se aos saberes médicos.

Considerada uma das mais importantes causas de morbimortalidade do mundo - sendo um dos mais prevalentes fatores de risco para o desenvolvimento de doença arterial coronariana, acidente vascular cerebral, doença vascular periférica, insuficiência renal e insuficiência cardíaca congestiva a HAS destaca-se no contexto familiar como uma experiência de difícil adaptação, pois seu controle requer mudanças de comportamento que vão desde os hábitos alimentares, até o estilo de vida não só da pessoa hipertensa, como de toda a família. ${ }^{2}$

Seguindo a lógica dessa narrativa e do incentivo de envolver a família no cuidado da pessoa hipertensa, cabe enfatizar que da convivência entre os membros da família emerge a condição primeira para detectar sinais de anormalidade no estado de saúde destes, assim como alterações no curso de uma doença. A participação ativa do familiar no cuidado se apresenta como um papel significativo no controle dos fatores de risco da HAS, mormente os relacionados à hereditariedade, contribuindo concretamente para tratamento da pessoa hipertensa.
O fator adaptação é ainda responsável pela enorme resistência que os profissionais encontram para a adesão do hipertenso ao tratamento. Percebese que a não-adesão é o grande entrave no controle da doença, o que justifica sua existência ser encarada como um problema de saúde pública. Investimentos nesse sentido vêm se tornando relevantes, mas a solução para este problema enfrenta ainda o desafio de envolver conjuntamente no tratamento a pessoa hipertensa e sua família. ${ }^{3}$

Reconhecendo que a concepção de saúde e de doença dos indivíduos é histórica e culturalmente formada - e que mudar essa concepção requer um (re)aprendizado, que também necessita somar história na vida da pessoa para reconstruir seus conceitos, inclusive acerca do que é ter saúde - considera-se que a educação que se volte para formar juízo acerca da práxis do cuidado do hipertenso no seio familiar deve despertar atenção, ensejando que a necessidade de investigação responda aos questionamentos: Quais saberes acerca da HAS vêm permitido elaborar a educação informal (e formal) no seio familiar? Como esses saberes do familiar contribuem para a adesão da pessoa hipertensa ao tratamento? Com base nesses questionamentos, optou-se pelo estudo com o objetivo de analisar o saber do familiar na adesão da pessoa hipertensa ao tratamento.

\section{METODOLOGIA}

Estudo exploratório-descritivo realizado na Liga de Hipertensão Arterial do Hospital de Messejana (LHAHM) - instituição pública do SUS, localizada em Fortaleza-CE. Na liga é desenvolvido um programa de hipertensão arterial pela equipe multiprofissional, cujo objetivo é o de reduzir a morbimortalidade por agravos cardiovasculares e cerebrovasculares associados à HAS, integrando e/ou reintegrando o usuário hipertenso à sociedade.

No momento da pesquisa 1600 pessoas estavam cadastradas na LHAHM, constituindo uma amostra de 400 familiares, previamente indicados pelas próprias pessoas hipertensas como aqueles que mais participavam do tratamento, e que concordaram em ser incluídos no estudo. Considerouse como familiares, aqueles oriundos de famílias extensas, como nucleares.

A coleta de dados ocorreu durante três meses, através de entrevista, utilizando um roteiro estruturado contendo dados sócio-demográficos, vínculo familiar e conhecimento do familiar sobre a HAS e 
o tratamento. Em seguida, organizaram-se os dados no programa Statistical Package for Social Science-SPSS (versão 13), representando-os em tabelas e quadros, que foram analisados com base nas informações dos familiares, nos pressupostos da educação popular em saúde e na literatura previamente selecionada.

Vale salientar que a pesquisa foi desenvolvida em respeito à Resolução 196/964 da Comissão Nacional de Ética em Pesquisa (CONEP) e que os dados foram coletados após assinatura do Termo de Consentimento Livre e Esclarecido e a emissão do parecer favorável do Comitê de Ética do Hospital de Messejana, Ministério da Saúde (MS), Sistema Único de Saúde (SUS), Secretaria de Saúde do Estado (SESA), com o número 262/05.

\section{RESULTADOS E DISCUSSÃO}

\section{Caracterização sócio-demográfica dos familiares}

De acordo com a Tabela 1, 206 (51,5\%) familiares eram filhos, 108 (27\%) cônjuges e 86 (21,5\%) tinham outro tipo de vínculo familiar (tios, pais, irmãos, sobrinhos, netos, avôs, primos, genros, cunhados, noras e sogros). Assim, evidencia-se que a participação do familiar não foi o fator determinante para a participação, embora concordemos com alguns autores ao afirmarem que, quanto maior o vínculo afetivo envolvido na relação, maior será a participação do familiar na provisão do cuidado. ${ }^{5-6}$

Tabela 1 - Distribuição dos familiares segundo a cor, a idade e o vínculo com a pessoa hipertensa. Fortaleza-CE, 2005.

\begin{tabular}{|c|c|c|c|c|c|c|c|c|c|c|c|}
\hline \multirow[b]{3}{*}{ Idade(anos) } & \multicolumn{3}{|c|}{ Filho } & \multicolumn{3}{|c|}{ Cônjuge } & \multicolumn{3}{|c|}{ Outros } & \multicolumn{2}{|c|}{ Total } \\
\hline & \multicolumn{9}{|c|}{ Cor } & & \\
\hline & B & $P$ & $\mathbf{N}$ & B & $\mathbf{P}$ & $\mathbf{N}$ & B & $\mathbf{P}$ & $\mathbf{N}$ & $f$ & $\%$ \\
\hline $20-29$ & 27 & 48 & 3 & 1 & 2 & - & 5 & 11 & 1 & 98 & 24,5 \\
\hline $30-39$ & 23 & 31 & 1 & 6 & 3 & 2 & 3 & 7 & 1 & 77 & 19,2 \\
\hline $40-49$ & 11 & 37 & 4 & 9 & 12 & 4 & 13 & 9 & 1 & 100 & 25,0 \\
\hline $50-59$ & 4 & 14 & 1 & 10 & 18 & 3 & 4 & 8 & 2 & 64 & 16,0 \\
\hline $60-69$ & 1 & 1 & - & 9 & 20 & 1 & 6 & 4 & 1 & 43 & 10,8 \\
\hline $70-79$ & - & - & - & 1 & 7 & - & 2 & 5 & 3 & 18 & 4,5 \\
\hline Total & 66 & 131 & 9 & 36 & 62 & 10 & 33 & 44 & 9 & 400 & 100,0 \\
\hline
\end{tabular}

* B-Branca; P-Parda; N-Negra

Segundo a Tabela 1, houve predomínio da faixa etária de 40 a 49 anos, com $100(25 \%)$ pesquisados, seguido de $98(24,5 \%)$ entre 20 e 29 anos de idade e de $77(19,2 \%)$ entre 30 e 39, contra $18(4,5 \%)$ entre 70 e 79 anos. Quanto à cor, a maioria eram pardos, e a minoria negros. Logo, constata-se que indivíduos negros e com idade acima de 40 anos, filhos de pessoas hipertensas de cor negra, apresentavam fatores de risco para se tornarem hipertensos, o que corrobora com os achados do estudo em que se percebeu a ausência da preocupação quanto à prevenção da HAS nos próprios familiares.

Esse fato nos leva a refletir sobre a ausência da prevenção da doença hereditária, como é o caso da HAS. Sabemos que as experiências empíricas subsidiam as experiências seguintes no decorrer do processo saúde-doença, e que contribuem para a adoção de determinadas posturas diante de situações de risco. No entanto, não detectamos nenhum ou quase nenhum indício de apreensão dos familiares quanto ao desenvolvimento da HAS.

A história familiar é um dos principais fatores de risco para o surgimento da HAS, ressaltando que a doença é mais grave no negro que no branco. ${ }^{7}$ Acrescenta-se, ainda, que obesidade, tabagismo, dieta rica em sódio e pobre em cálcio, maus hábitos de vida, baixo nível educacional, estresse emocional e uso de drogas, são fatores que predispõem seu aparecimento. Contudo, se houver uma modificação dos hábitos de vida, bem como redução dos fatores de risco, isso pode influenciar positivamente na prevenção da doença. 
Tabela 2 - Distribuição dos familiares segundo ocupação, escolaridade e renda mensal. FortalezaCE, 2005.

\begin{tabular}{|c|c|c|c|c|c|c|c|c|c|c|c|c|c|c|c|c|c|}
\hline \multirow{3}{*}{ Renda mensal*** } & \multicolumn{5}{|c|}{ Aposentado } & \multirow{2}{*}{\multicolumn{5}{|c|}{$\begin{array}{l}\text { Prendas do lar } \\
\text { Escolaridade }^{\star *}\end{array}$}} & \multicolumn{5}{|c|}{ Outras* } & \multicolumn{2}{|c|}{ Total } \\
\hline & \multirow[b]{2}{*}{ A } & \multirow[b]{2}{*}{ B } & \multirow[b]{2}{*}{ C } & \multirow[b]{2}{*}{ D } & \multirow[b]{2}{*}{$E$} & & & & & & & & & & & & \\
\hline & & & & & & A & B & C & D & $E$ & A & B & $\mathrm{C}$ & $\mathrm{D}$ & $E$ & $f$ & $\%$ \\
\hline$<1$ & - & - & 2 & - & - & - & 1 & 13 & 9 & - & - & 1 & 8 & 20 & - & 54 & 13,5 \\
\hline $1-2$ & 5 & 11 & 15 & 5 & - & - & 8 & 38 & 11 & - & 5 & 6 & 61 & 80 & 9 & 425 & 63,5 \\
\hline 3 ou mais & 2 & 1 & 1 & 5 & 1 & - & 1 & 10 & 10 & - & - & 11 & 15 & 32 & 13 & 92 & 23,0 \\
\hline Total & 7 & 12 & 18 & 10 & 1 & - & 10 & 61 & 30 & - & 5 & 8 & 84 & 132 & 22 & 400 & 100,0 \\
\hline
\end{tabular}

* Outras: Vendedor, empregada doméstica, agricultor, auxiliar de produção, motorista, professor.

** Escolaridade: A-Analfabeto; B-Alfabetizado; C-Ensino fundamental; D-Ensino médio; E-Ensino superior.

*** Em salário mínimo.

$\mathrm{Na}$ Tabela 2, a maioria dos entrevistados cursou até o ensino médio e um pequeno número havia cursado ensino superior. Cabe ressaltar esse dado, pois a partir dele novas estratégias podem ser traçadas, buscando que o familiar tenha uma maior participação no tratamento e possa expressar seu saber popular e empírico, partindo do princípio de que a escolaridade maior favorece a compreensão sobre a doença e o cuidado. Conseqüentemente, a instrução se torna um elemento relevante e contribuinte na busca da adesão da pessoa hipertensa ao tratamento.

Quanto à renda mensal, 63,5\% dos familiares informou receber de um a dois salários mínimos e 13,5\%, abaixo de um. Esse fato confirma que a amostra do estudo faz parte de uma classe social desprovida de recursos financeiros significativos, e dependente do SUS.

Entre os familiares participantes do estudo, constatou-se 218 (57,2\%) oriundos de família nuclear, que tinham idade acima de 40 anos para o homem e de 50 anos para a mulher, os achados constituíam riscos para o desenvolvimento da HAS. Logo, este fato deveria despertá-los para a adoção de práticas preventivas desse agravo.

Vale esclarecer que a prevalência da HAS aumenta com a idade, o que deveria servir de incentivo para que os familiares tivessem uma maior preocupação quanto à prevenção da doença, tendo em vista o caráter hereditário somado à idade, hábitos inadequados e sedentarismo, entre outros, por serem considerados importantes fatores de risco desse agravo.
Todavia, tem havido muitas discussões sobre a história familiar, HAS e os fatores de risco ambiental (ou de estilo de vida); ou seja, dependendo do meio ambiente em que a pessoa vive e da sua exposição a outros fatores de riscos associados, o indivíduo é mais ou menos propenso a desenvolver ou não a doença. ${ }^{8}$

Os dois tipos de famílias existentes são nuclear e extensa ou ramificada. A primeira se caracteriza pelo vínculo direto, o de pai, mãe e filhos. Enquanto que a segunda é definida por diferentes gerações vivendo num mesmo ambiente familiar, incluindo também aqueles membros que mantêm laços afetivos estreitos 9 . Sendo assim, encontramos nas informações obtidas com o estudo, a predominância da família nuclear, em que há também o(s) irmão(s) com vínculo direto, bem como àquela família extensa, na qual existem diversos vínculos.

\section{Análise do saber do familiar sobre a HAS e o tratamento}

Segundo Tabela 3, para 274 (68,5\%) familiares, a HAS significava pressão alta, pressão arterial descontrolada e doença na família. Para 339 $(84,7 \%)$, os fatores de risco para HAS incluíam ingesta inadequada de sal, uso de gordura animal, estresse, álcool, obesidade, tabagismo e sedentarismo. Percebe-se, assim, um saber elementar acerca dos fatores de risco para doença hipertensiva. Entretanto, há outros elementos que devem ser destacados nesse contexto. Incluem-se como fatores de risco as características constitucionais, como sexo, idade, etnia e antecedentes pessoais. 
Tabela 3 - Distribuição dos familiares segundo o saber sobre a HAS, os fatores de risco, as complicações, a gravidade e a cronicidade. Fortaleza-CE, 2005.

\begin{tabular}{lcccc}
\hline Saber & \multicolumn{2}{c}{ Sim } & \multicolumn{2}{c}{ Não } \\
& $\mathbf{n}$ & $\mathbf{0}$ & $\mathbf{n}$ & \% \\
\hline Gravidade & 388 & 97,0 & 12 & 3,0 \\
Complicações & 343 & 85,7 & 57 & 14,3 \\
Fatores de risco & 339 & 84,7 & 61 & 15,3 \\
Cronicidade & 306 & 76,5 & 94 & 23,5 \\
Concepção sobre HAS & 274 & 68,5 & 126 & 31,5 \\
\hline
\end{tabular}

$\mathrm{Na}$ literatura, fator de risco representa a probabilidade de pessoas se exporem a certas condições que as levem a adquirir subseqüentemente uma determinada doença, incluindo aí a susceptibilidade que alguns indivíduos têm frente esta exposição. Sendo assim, os fatores de risco na gênese da HAS, compreendem: a idade, cuja prevalência fica em torno de $7 \%$ para crianças, $20 \%$ para os adultos e $65 \%$ para os idosos; o sexo, cuja mulher até antes da menopausa, devido à produção de estrogênio, em que se acredita que exerça a função protetora sobre o sistema cardiovascular feminino, é menos acometida que o homem, porém, a partir dessa etapa de vida, ela se iguala ao homem; a raça, em que há uma predominância nos negros, bem como de maiores complicações; os antecedentes familiares, os quais quando associados a outros fatores, contribuem para o desenvolvimento da morbidade; a alimentação, que se reflete no uso ou não de alimentos saudáveis, com ênfase no consumo excessivo de sal e gorduras animais; a obesidade, um fator em potencial para o desenvolvimento da doença; o estresse, pela estimulação excessiva do sistema nervoso simpático, aumentando os níveis pressóricos; o tabagismo, devido ao aumento da pressão arterial pela presença da nicotina; o álcool, que age na musculatura cardíaca; o sedentarismo, associado ao atual estilo de vida da população; e o uso de contraceptivos orais, pela ação sobre o sistema cardiovascular da mulher. ${ }^{8}$

Cerca de 343 (85,7\%) entrevistados associaram as complicações com acidente vascular cerebral, infarto agudo do miocárdio e miocardiopatia dilatada. E a gravidade da HAS, para $388(97,0 \%)$ familiares correspondia ao que é de fato, pois admitiam o risco de complicações graves que levavam à invalidez ou à morte iminente.
Trezentos e seis $(76,5 \%)$ entrevistados reconheceram a impossibilidade de cura da HAS, o que denota um relativo saber acerca da cronicidade. Dessa forma, acrescenta-se que por ser uma doença crônico-degenerativa, seu tratamento é contínuo e ininterrupto, sendo, talvez, esse o motivo pelo qual os familiares reconheciam a ausência de cura.

Vale ressaltar que a maioria dos familiares afirmou existirem manifestações clínicas da HAS, contrariando o que diz a literatura quanto a assintomatologia da doença. Entre as respostas, predominaram dor de cabeça, seguida da dor na nuca.

Acredita-se que é difícil para o familiar aceitar que a HAS é uma doença assintomática, pois ele relaciona a dor de cabeça com o aumento dos níveis pressóricos. Entretanto, no universo das doenças de história natural prolongada, com multiplicidade de fatores de risco complexos, interações de fatores etiológicos e biológicos conhecidos e/ou desconhecidos, longo período de latência e longo curso assintomático, curso clínico em geral prolongado e constante, manifestações clínicas com períodos de clemência e exacerbação, evolução para graus variados de incapacidades ou para morte, a HAS se destaca pela dimensão de suas múltiplas expressões. ${ }^{10}$

Conforme a Tabela 4, a maioria dos familiares sabia como condutas o uso adequado de sal $(90,2 \%)$ e uso adequado de gordura vegetal (62\%); no entanto, o maior número de condutas saudáveis eram desconhecidas, tais como, o uso de adoçantes dietéticos (86,5\%), a ingesta adequada de café $(82,3 \%)$, o peso controlado (80,7\%), a abstenção do álcool $(71,5 \%)$, a abstenção do tabaco (68\%), a preferência por vegetais $(67 \%)$, e por carnes brancas $(63,2 \%)$ e o gerenciamento do estresse (56\%). 
Tabela 4 - Distribuição dos familiares, segundo o saber sobre as condutas do tratamento. FortalezaCE, 2005.

\begin{tabular}{lcccc}
\hline Condutas do tratamento & \multicolumn{2}{c}{ Sim } & \multicolumn{2}{c}{ Não } \\
& $\mathbf{n}$ & $\mathbf{0}$ & $\mathbf{n}$ & $\%$ \\
\hline Uso regular do medicamento & 364 & 91,0 & 36 & 9,0 \\
Uso adequado de sal & 361 & 90,2 & 39 & 9,8 \\
Uso de gordura vegetal & 248 & 62,0 & 152 & 38,0 \\
Gerenciamento do estresse & 176 & 44,0 & 224 & 56,0 \\
Preferências por carnes brancas & 147 & 36,8 & 253 & 63,2 \\
Exercício físico regular & 144 & 36,0 & 256 & 64,0 \\
Preferências por vegetais & 132 & 33,0 & 268 & 67,0 \\
Abolição do tabaco & 128 & 32,0 & 272 & 68,0 \\
Abolição do álcool & 114 & 28,5 & 286 & 71,5 \\
Peso adequado & 77 & 19,3 & 323 & 80,7 \\
Ingestão adequada de café & 71 & 17,7 & 329 & 82,3 \\
Uso de adoçantes dietéticos & 54 & 13,5 & 346 & 86,5 \\
\hline
\end{tabular}

Tanto o familiar como a pessoa hipertensa, desconheciam as condutas do tratamento. Em estudo realizado sobre o conhecimento do cliente hipertenso quanto ao tratamento, estes conheciam de forma inadequada os riscos da HAS não con- trolada. ${ }^{3}$ Este fato favorece o aumento dos casos de complicações cardiovasculares e o aumento dos custos sociais com as aposentadorias precoces por invalidez, licença para tratamento de saúde, absenteísmo ao trabalho, etc.

Tabela 5 - Distribuição dos familiares segundo o saber acerca do esquema medicamentoso. FortalezaCE, 2005.

\begin{tabular}{lcccc}
\hline Esquema medicamentoso & \multicolumn{2}{c}{ Sim } & \multicolumn{2}{c}{ Não } \\
& $\mathbf{n}$ & \% & $\mathbf{n}$ & \% \\
\hline Tipo de drogas anti-hipertensivas & 264 & 66,0 & 136 & 34,0 \\
Número de tomadas diárias & 220 & 55,0 & 180 & 45,0 \\
Concentração diária & 90 & 22,5 & 310 & 77,5 \\
Desconfortos causados pelos medicamentos & 46 & 11,5 & 354 & 88,5 \\
Efeitos adversos ou colaterais & 07 & 1,8 & 393 & 98,2 \\
\hline
\end{tabular}

Observa-se que na Tabela 5, 264 (66\%) entrevistados sabiam o tipo de droga indicada para o hipertenso; entretanto, apenas 90 (22,5\%) familiares sabiam a concentração diária da droga ingerida. Dentre os pesquisados, $220(55 \%)$ referiram o número de tomadas e 393 (98,2\%) desconheciam os efeitos colaterais que a droga poderia gerar. Cerca de 364 (91\%) sabiam do uso regular e $354(88,5 \%)$ nada informaram sobre os possíveis desconfortos.
Dessa maneira, percebe-se que o saber do familiar sobre o esquema medicamentoso indicado para o hipertenso era elementar e se restringia basicamente ao nome da droga, embora em muitos casos de esquema complexo de medicação, só soubesse um ou dois nomes. Isso reflete uma falha importante da participação desse familiar, que referia atuar no tratamento sem que, no entanto, conhecesse o esquema medicamentoso indicado, favorecendo a 
não-adesão do hipertenso ao tratamento. Portanto, é imprescindível que a participação do familiar seja completa e responsável.

Para cuidar (aqui se usa participar) é necessário desvelo, solicitude, diligência, zelo, atenção e bom trato, e se há o compromisso para tal ação, há de se provocar a preocupação, inquietação e senso de responsabilidade em quem se predispõe, porque o individuo que assume esse papel deve-se sentir envolvido e ligado afetivamente ao outro que recebe seu cuidado e que, de alguma forma, sente-se grato. ${ }^{11}$

A história humana tem sempre contado com o cuidar, sendo este uma forma de viver e de se relacionar. $\mathrm{Na}$ saúde, ele busca a cura, tarefa concedida aos profissionais de saúde, em destaque o enfermeiro, mas que se permeia no seio familiar de maneira inata, variando, entretanto, de acordo com a cultura do meio onde é projetado. ${ }^{12}$

O conhecimento sobre a doença e o tratamento da HAS, influencia diretamente na adesão a esse. Conseqüientemente, a escolaridade atua como fator determinante neste fato, pois as informações só são assimiladas a partir do momento em que haja capacidade cognitiva para isso. O contrário é recíproco, ou seja, se não há capacidade cognitiva não haverá apreensão das informações, não ocorrendo também, compreensão sobre a importância de aderir ao tratamento. ${ }^{13}$

Entretanto, esse estudo mostra que o conhecimento foi regular mesmo em analfabetos ou naqueles em que a escolaridade é baixa, alfabetizados, e que apresentaram um relativo saber sobre as modalidades do tratamento indicado para a pessoa hipertensa, provando a existência da condição de envolver intrinsecamente o familiar no tratamento dessa pessoa.

Tabela 6 - Distribuição dos familiares segundo as terapias complementares para o controle da HAS na pessoa hipertensa. Fortaleza-CE, 2005.

\begin{tabular}{lcc}
\hline Terapias complementares & $\mathbf{n}$ & $\%$ \\
\hline Chás & 377 & 94,2 \\
Outras & 28 & 7,0 \\
Sucos & 19 & 4,7 \\
Crença/prática religiosa & 13 & 3,2 \\
\hline
\end{tabular}

De acordo com o Tabela 6, 377 (94,2\%) familiares utilizavam chás dos variados tipos - capim- santo, cidreira, colônia, erva doce, alho; 19 (4,7\%) indicaram os sucos de maracujá, chuchu, berinjela, etc; $13(3,2 \%)$ apelavam para crença através de rituais religiosos; e 28 (7,0\%) adotavam outras terapias, produzidas com vegetais, tais como garrafada de carnaúba, água de oiticica, água da berinjela, água com limão, talo do alface, chuchu cozido, cana-naninha, cupim de cajueiro, folha de laranja, semente de maracujá triturada, aguardente alemã, água de alho, água de pepino, água de chuchu, casca da melancia, e folha de carambola.

Crenças populares e recursos não convencionais, utilizados na solução de problemas de saúde, configuram-se para a população em geral como fatores extremamente ligados a aspectos sócio-culturais, por isso devem ser considerados como relevantes quando se avalia o indivíduo como um ser integral, pertencentes a um processo histórico. ${ }^{14}$

"Decorre da importância da educação em saúde buscar explorar a forma como a doença é elaborada culturalmente, tendo como horizonte levar os sujeitos ao reconhecimento desta como algo anômalo, vindo assim a favorecer comportamentos de recusa, negação e remoção da enfermidade - fenômeno que pode ser designado como de 'desnaturalização' da doença, segundo o qual a doença é extraída de um contexto em que é vista como normal, natural. Enquanto educador em saúde, esta é uma tentativa de abdicar de um poder fictício e permitir ser seduzido pelo outro para criar novas ou outras representações. Aprender a relativizar os conhecimentos e permitir trocas possíveis com os sujeitos com os quais se relaciona em seu processo saúde-doença". ${ }^{15: 200}$

\section{CONCLUSÃO}

O indivíduo é dono de um saber único e particular relacionado intimamente com sua história de vida e suas experiências, construindo conceitos e concepções para adotar uma posição diante das situações. Seu saber não é considerado científico, o que não significa que não tenha valor. Pelo contrário, todos têm saber, todos têm valor. O valor que é dado ao saber individual é aquele que traz o cotidiano implícito nas relações humanas, fortalecendo os vínculos entre as pessoas e promovendo o cuidado entre elas.

Evidenciou-se entre os participantes do estudo que a maioria que se destacava no cuidado eram filhos e cônjuges, na faixa etária de 40 a 49 
anos. Com relação ao saber sobre a HAS e seu tratamento, pôde-se constatar que era elementar, ou seja, eles apresentaram algumas noções sobre a doença e o tratamento. Informaram o uso excessivo de sal, gordura animal e de álcool como fatores de risco para a HAS; e associaram como complicações o infarto agudo do miocárdio, acidente vascular cerebral e miocardiopatia dilatada. Todavia, admitiu-se a cefaléia como uma manifestação clínica clássica da HAS. Como condutas terapêuticas, indicaram somente o uso regular do medicamento, redução do sal e abstenção de gordura. Contudo, em relação ao tratamento medicamentoso, a maioria conhecia o tipo de droga e sabia o número de tomadas, porém ignorava os efeitos terapêuticos e adversos da droga.

Outro achado do estudo vinculado ao saber popular foi a adoção de terapias complementares, entre elas predominou a indicação de uso de chás para o controle da pressão arterial entre os participantes. Traz-se à tona a educação popular como cenário maior do nosso estudo, a fim de expô-la como elemento fundamental para apresentar o saber (re)construído pela família através das experiências vivenciadas no cuidado diário com seus familiares hipertensos, e como primeira instituição do cuidado humano.

Os matizes da cultura e das relações de proximidade entre os sujeitos envolvidos vão delineando as especificidades nos modos de cuidar. Essas especificidades culturais precisam ser consideradas em todos os grupos sociais, buscando alternativas de cuidados mais aproximadas entre o cuidado profissional e o popular - algumas vezes mantendo, outras negociando e outras remodelando o cuidado cultural. ${ }^{15}$

Então, diante desses achados, sugere-se um (re)planejamento de estratégias educativas em saúde com vistas a capacitar não somente os usuários hipertensos, mas também os familiares no controle da HAS, resgatando o saber dessas pessoas, bem como, valorizando a sua cultura.

\section{REFERÊNCIAS}

1 Stotz EN. A cultura e o saber: linhas cruzadas, pontos de fuga. In: Vasconcelos EM. A saúde nas palavras e nos gestos: reflexões da rede de educação popular e saúde. São Paulo (SP): Hucitec; 2001.p.88-93.
2 Andrade JP. Aspectos epidemiológicos da aderência ao tratamento da hipertensão arterial sistêmica. Arquivo Bras Cardiol. 2002 Out.; 79 (4):375-84.

3 Péres DS, Magna JM, Viana LA. Portador de hipertensão arterial: atitudes, crenças, percepções, pensamentos e práticas. Rev. Saúde Públ. 2003 Out; 37 (5): 635-42.

4 Ministério da Saúde (BR), Conselho Nacional de Saúde, Comissão Nacional de Ética em Pesquisa. Resolução No. 196, de 10 de outubro de 1996: diretrizes e normas regulamentadoras de pesquisas envolvendo seres humanos. Brasília (DF): O Conselho; 1996.

5 Santos ZMSA, Frota MA, Cruz DM, Holanda SDO. Adesão do cliente hipertenso ao tratamento: análise com abordagem interdisciplnar. Texto Contexto Enferm. 2005 Jul-Set; 14 (3): 332-40.

6 Szymanski H. Teorias e "teorias" de famílias. In: Carvalho MCB. A família contemporânea em debate. São Paulo (SP): EDUC/Cortez; 2003. p.45-54.

7 Oigman W. Avaliação clínico-laboratorial do paciente hipertenso objetivando a decisão terapêutica. In: Pierin AMG. Hipertensão arterial: uma proposta para o cuidar. São Paulo (SP): Manole; 2004. p.23-35.

8 Siqueira FPC, Veiga EV. Hipertensão arterial e fatores de risco. Enferm. BR. 2004 Maio; 3 (2): 101-6.

9 Elsen I, Marcon SS, Santos MR, organizadores. O viver em família e sua interface com a saúde e a doença. Maringá (PR): EDUEM; 2002.

10 Wetzel Júnior W, Silveira MPT. Hipertensão arterial: um problema de todos. Rev. Nursing 2005 Fev; 81 (8): 70-5.

11 Boff L. Saber cuidar: ética do humano, compaixão pela terra. Petrópolis (RJ): Vozes; 2000.

12 Waldow VR. Cuidado humano: o resgate necessário. Porto Alegre (RS): Sagra Luzzato; 2001.

13 Pierin AMG, Gusmão JL, Carvalho LVB. A falta de adesão ao tratamento como fator de risco para hipertensão arterial. Rev. da Socied. Brasil. Hipertensão 2004 Jul; 7 (3): 100-3.

14 Gazzinelli MF, Gazzinelli A, Reis DC, Penna CMM. Educação em saúde: conhecimentos, representações sociais e experiências da doença. Cad. Saúde Públ. 2005 Jan-Fev; 21 (1): 200-6.

15 Budó MLD, Saupe R. Modos de cuidar em comunidades rurais: a cultura permeando o cuidado de enfermagem. Texto Contexto Enferm. 2005 AbrJun; 14 (2): 177-85. 